\title{
Lung Deformation Estimation with Non-rigid Registration for Radiotherapy Treatment
}

\author{
Vlad Boldea ${ }^{1}$, David Sarrut ${ }^{1,2}$, and Sebastien Clippe ${ }^{1,2}$ \\ 1 LIRIS, Universite Lumiere Lyon 2, \\ 5 Av. Pierre Mendes-France - 69676 Bron, France \\ 2 Radiotherapy Department, Centre Leon Berard, \\ 28 rue Laennec - 69353 Lyon, France
}

\begin{abstract}
A main challenge in radiotherapy is to precisely take into account organs deformation and motion in order to adapt the treatment to each patient. This is particularly important in lung cancer where breathing leads to large displacements. In this work, breath holding techniques (with Active Breath Control device - ABC) were used to reduce movements during treatment. We study thorax and lung deformation between different CT scans acquired at same and different breathing stages. We developed non-rigid registration tools to evaluate for each patient the reproducibility of $\mathrm{ABC}$ and to extract motion information for subsequent dosimetric and modeling studies. First results show that ABC has a good reproducibility, that vector fields can be used to detect pathological situations and that deformations due to breathing can be estimated.
\end{abstract}

\section{Medical Context}

Radiotherapy is one of the three main cancer treatment modalities, together with surgery and chemotherapy. It consists in delivering a maximum dose of $\mathrm{X}$-rays to the tumor while sparing surrounding normal tissue. Physicians and physicists begin to establish a Radiotherapy Treatment Planning (RTP) defining the number of beams, their size, their shape, their tilt and the beam energy. This RTP is computed from a computed tomography (CT) 3D scan of the patient. To be efficient, the treatment is fractionated in several sessions (between 30 and 40). During each session, the patient is setup on the table couch and the prescribed dose is delivered according to the RTP.

A major difficulty is the daily patient setup reproducibility. Computed-aided methods using control images are proposed (see for example [12]). Another major difficulty is organ motion and deformation, particularly in the thorax because of patient breathing. Currently in routine, large margins are added to the target volume. A main challenge in radiotherapy is to take into account more precisely this motion and to adapt the treatment to each patient.

One way to do is to use breath hold devices in order to immobilize the patient during treatment. In our institution, two clinical studies which use ABC (Active Breath Control, see [3]) have begun. ABC allows to block airflow under patient control at a preset lung volume during either inspiration or expiration, thereby 
temporarily immobilizing breathing motion. The duration of the active breathhold is set such that the patient can comfortably maintain this breath holding. Radiation will be turned on and off during this period. This system presents two main interests. First, it leads to less tumor motion during irradiation. Secondly, in inspiration, it increases the total lung volume, it decreases lung density and thus it decreases the percentage of irradiated healthy lung [34].

The goal of this work is to study thorax and lung deformation between different CT scans acquired at same and different breathing stages. We developed tools to evaluate for each patient the reproducibility of ABC and to extract motion information for subsequent dosimetric and modeling studies. Next section presents non-rigid registration algorithm used for motion detection. Section 3 presents materials and methods. Section 4 shows some experiments and results.

\section{Monomodal 3D Non-rigid Registration}

\subsection{State of the Art}

The main goal of image registration techniques is the mapping of two or several images. Many studies (see for example [56 for surveys) have focalized on algorithms for non-rigid registration especially for medical images where the information of organ deformation is often vital for patient treatment. Methods can be divided in two classes : sparse and dense. In the first one, deformation is evaluated from a few set of control points with a deformation model such as thin-plate spline 78 . In the second one, a displacement vector is computed at each point leading to a dense deformation field. Here, we focus on monomodal dense non-rigid registration.

In monomodal registration, the assumption is that there is no change in intensity between a voxel of an image and its corresponding in the other. Dense methods consist in a tradeoff between voxels matching and vector field regularization. Continuum mechanics represents an inspiration source for medical image registration. One image is a deformable body and the other is the deformed (reference) body. Similarity measure may be assimilated to the force field. Vector field is regularized according to the equilibrium equation [9] or to alternative regularization energies 1011. Regularization can be applied on the velocity field, leading to fluid registration [129 allowing large deformation recovery. Other methods are based on optical flow theory [13 14 (under small displacements hypothesis) or diffusion theory [1516]. Thirion [17] proposed a well known method related to optical flow, called "demons" which will be described latter.

Regarding lung deformation estimation, Fan [18] introduces a model for dense lung warping between different breathing stages. It is based on interpolation of a sparse vector field which is generated from manually selected control points and surface mapping. The considered regularization is a linear combination of optical flow constraint and anisotropic smoothness constraint derived from continuum mechanics. 


\subsection{Dense Vector Field Estimation}

We use the "demons" algorithm of Thirion [17, modified by Cachier [19] because it allows to retrieve small and large dense deformation fields. Moreover in our case, the intensity conservation assumption is verified (images are monomodal and are acquired in the same conditions). It is an iterative process consisting of two steps: pairing and regularizing. At each iteration $i$, at each voxel $\boldsymbol{x}$, a displacement vector $u_{i}(\boldsymbol{x})$ is evaluated as eq. 1 I, J denotes the images, $T_{i}$ denotes the transformation at iteration $i, \nabla$ denotes the gradient operator. The deformation is evaluated in the gradient direction and it is directly proportional to the intensity difference between $I(\boldsymbol{x})$ and $J(T(\boldsymbol{x}))$. Cachier introduced the real parameter $\alpha(\alpha>0)$ in order to limit the displacement vector for small gradients: the norm is thus bounded by $1 /(2 \alpha)$.

$$
u_{i}(\boldsymbol{x})=\frac{I(\boldsymbol{x})-J\left(T_{i-1}(\boldsymbol{x})\right)}{\|\nabla I\|^{2}+\alpha^{2}\left(I(\boldsymbol{x})-J\left(T_{i-1}(\boldsymbol{x})\right)\right)^{2}} \nabla I
$$

Deformation field $T$ is obtained from $u$ by: $T_{i}=\operatorname{smooth}\left(T_{i-1}+u_{i}\right)$. At each iteration the resulting transformation is regularized by a 3D Gaussian filter implemented by Deriche recursive filters [20] which gives the advantage of a fast computation: the Gaussian filter is separable and the recursive implementation allows to have a fixed number of operations per displacement vector independently of the width of the filter. Displacements are bounded by $1 /(2 \alpha)$ at each iteration, but the fact that each iteration starts from the previously found $T_{i-1}$, can lead finally to larger displacements.

\section{Materials and Methods}

\subsection{Materials}

We used two sets of data. The first one consists in three 3D CT scans acquired with $\mathrm{ABC}$, where patients have been breath held at the same preselected phase in the breathing cycle (about $70 \%$ of the vital capacity). Six patients were included in this set (among 50 planned). In the second set patients have been breath held at three different stages (with different levels according to the patient ability). This set contains 2 patients (among 35 planned). Voxels values are encoded in 16 bits and expressed in Hounsfield units (related to density) in order to keep significant information for evaluating the deformation field inside the lungs.

\subsection{Methods}

Masks extractions. In order to evaluate points displacement only in regions of interest, we extracted two masks by thresholding and morphological tools. The first one removed from initial images the voxels not belonging to the patient (surrounding environment such that air, table). The second one considered voxels belonging to the lung and separated trachea and right and left lung. The procedure started with an automatic threshold [21], then we performed connected 
components labeling and several erosions and controlled dilatation operations in order to obtain voxels classification. The same automated procedure is applied on each image in order to obtain comparable results.

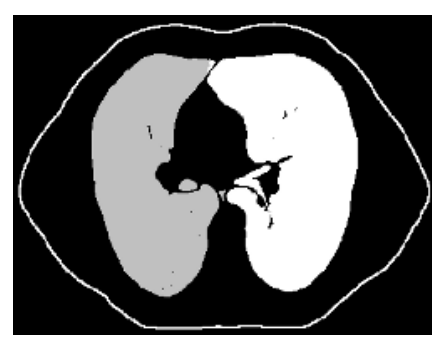

(a)

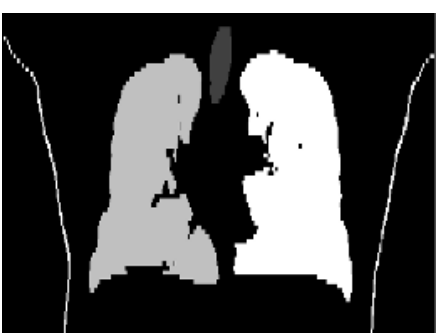

(b)

Fig. 1. Slices (axial (a) and coronal (b)) of segmented CT. Trachea, left and right lungs are displayed with different gray levels. Patient masks boundaries are displayed in white.

Demons's parameters. We observed that a good compromise between the convergence rate and the final error (sum of absolute differences) was obtained for $\alpha \in[0.5,0.65]$, leading to maximum vector displacement of $[0.77,1]$ voxel (by iteration, final displacement can be larger). Gaussian variance was fixed at 1.0. The stop criterion was fixed at 150 iterations. A multiresolution procedure was also used; images were downsampled by linear interpolation (the number of slices was preserved). We evaluated vector field at a resolution of $256 \times 256 \times 65(1.9$ $\mathrm{mm}$ in intra-plane direction and $5 \mathrm{~mm}$ inter-plane direction). Memory usage was about $200 \mathrm{MB}$, time speed was about fifteen minutes for a complete registration (without any optimization, on a Pentium $42.8 \mathrm{Ghz}$ ).

Pure deformation. Computed displacement between two scans is due to patient setup and organ motion. In order to evaluate only organ motion, we performed the following procedure. We considered that the patient setup displacement is reduced to a rigid transformation, which can be subtracted from the vector field obtained by the demons algorithm. We have experimented two methods to find the rigid transformation. In the first one, we evaluated it with a rigid registration algorithm 22] applied before demons's algorithm (the non-rigid algorithm started then from the founded rigid transformation). The second method estimated the transformation after the demons's algorithm. It consists in extracting from the vector field the closest rigid transformation. This is done by an optimization algorithm that minimize the sum of differences (eq. 2) where $\widetilde{R}$ denotes the global rigid deformation, $R(\boldsymbol{x})$ denotes the rigid deformation for the $\boldsymbol{x}$ point, $T(\boldsymbol{x})$ denotes the deformation vector (given by demons algorithm) at point $\boldsymbol{x}, d(a, b)$ is the distance between $a$ and $b, \Omega$ denotes the region of interest (which is defined as one of the masks previously described). Optimization is done with the Powell-Brent method [23]. 


$$
\widetilde{R}=\arg \min \sum_{\boldsymbol{x} \in \Omega} d(R(\boldsymbol{x}), T(\boldsymbol{x}))
$$

\section{Experiments and Results}

\subsection{Dataset 1: CT Hold at Same Breath Level}

Vector fields were used to assess reproducibility of breath holding and detect eventual errors. For each three images of a given patient, a vector field is computed for each couple of images (each image is alternatively reference and deformable image), leading to 6 evaluations per patient (36 vector fields). Figure 2 shows representative histograms of the displacement vector norms computed inside the patient (top figure) and inside the lung (bottom figure). All patients except one show small displacements (mean is $2.9 \mathrm{~mm}$, median is $2.3 \mathrm{~mm}$, standard deviation is $2.1 \mathrm{~mm}$; such statistics are computed with 30 vector fields). In this cases, breath holding was effective. However, patient 5 presents large displacements (mean is $6.4 \mathrm{~mm}$, median is $4.5 \mathrm{~mm}$, standard deviation is $5.8 \mathrm{~mm}$ ) due to a right lower lobe atelectasis (collapse of part of a lung by blockage of the air passages) which modified between acquisitions. In this case breath holding with ABC must not be used because of the abnormality of the lung behavior. So, vector field not only measures displacements but also can be used to detect pathological situations.

Several acquisitions showed large setup errors (translation is greater than $34 \mathrm{~mm}$ in the rigid transformation), but subtracting such setup errors allows to retrieve a pure measure of the deformation and to conclude that the breath holding was effective.

Thanks to the binary masks, lung volume measurements were also performed by counting the number of voxels inside trachea and left and right lung. Standard deviation of lung volumes is between 40 and $91 \mathrm{ml}$, except for the patient with atelectasis, where it is greater than $300 \mathrm{ml}$.

\subsection{Dataset 2: CT Hold at Different Breath Level}

Figure 3 shows subsampling (every $6 \mathrm{~mm}$ ) of the vector field in two slices (axial and sagittal). The vector field shows that patient's back is quasi-rigid, whereas thorax front part expenses. Main displacements concern the diaphragmatic cupola (vertical translation more than $30 \mathrm{~mm}$ ). Displacements inside the lung seem to be correctly estimated. There were few differences between the two methods used to compute the rigid transformation (mean difference less than 0.5 $\mathrm{mm}$ ), except for a patient with important increase in volume due to inspiration which leads the rigid registration algorithm to fail.

Several points have a negative Jacobian, which means that the deformation is locally non-invertible. This is due to the Gaussian regularization which do not guaranty that the deformation is a homeomorphism. However, there are no more than one hundred of points with negative Jacobian for dataset 1 and about 

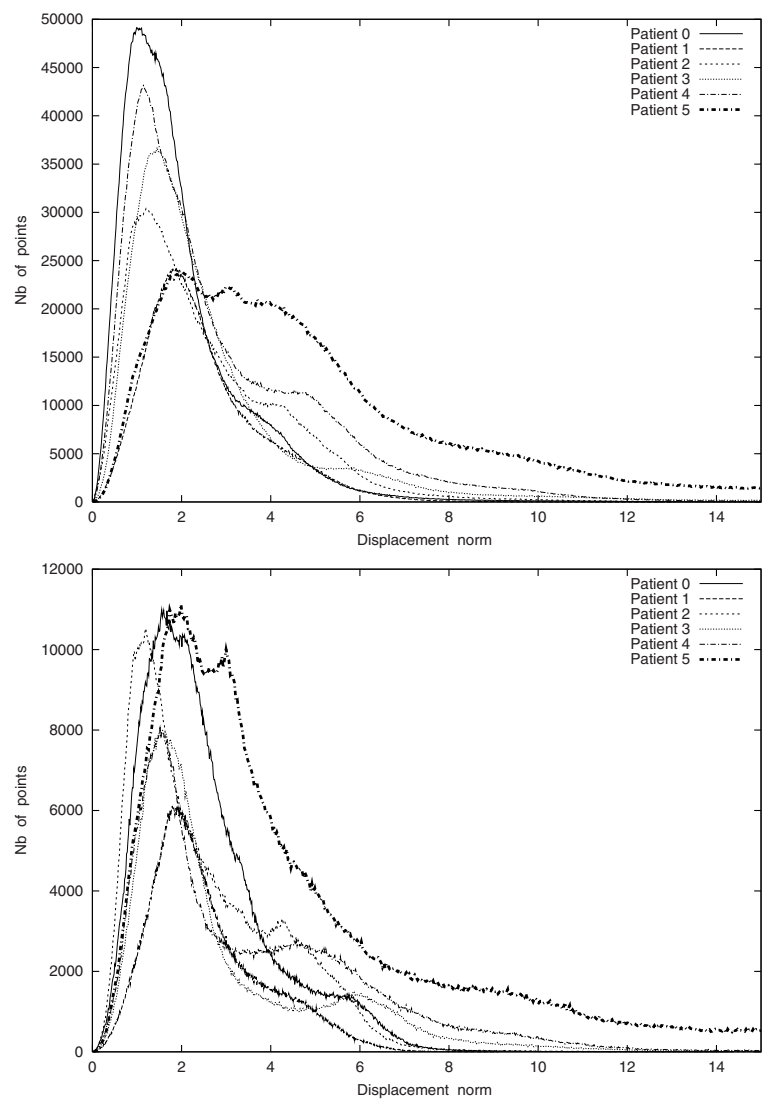

Fig. 2. Histogram of displacement norms inside patient (top) and lung (bottom). Patient 5 presents displacements larger than other patients. This is due to an atelectasis.

50000 for dataset 2 (1\% among more than 4 millions of points) for a resolution of $256 \times 256 \times 65(1.9 \mathrm{~mm}$ in intra-plane direction and $5 \mathrm{~mm}$ inter-plane direction).

\section{Conclusion}

In the quest to integrate motion and deformation information in radiation therapy, we have presented a study allowing to estimate points displacement into the thorax between CT scan acquired with breath hold. The proposed tool, a non-rigid registration based on demons algorithm, allows to extract useful information. By comparing vector fields between several acquisitions taken at same breath hold level, it is possible to quantify organs displacement in order to adapt margins for each patient in the treatment and to detect abnormal situations. Points displacements between images acquired at different breath hold level will 


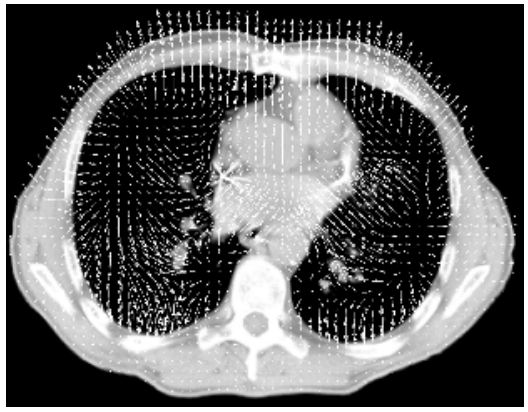

(a)

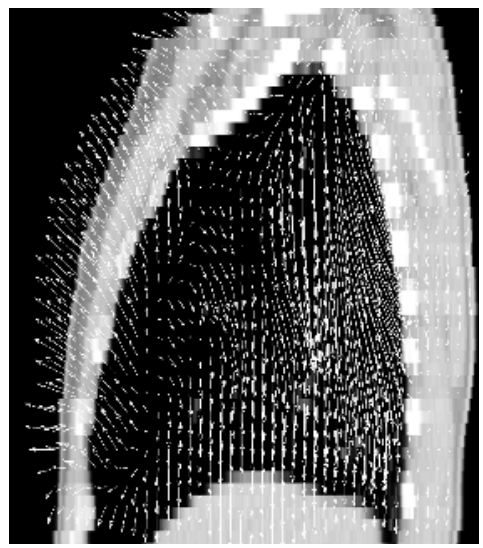

(b)

Fig. 3. Subsampled vector field overlaid with two slices (axial (a) and sagittal (b)) of a CT scan. Vectors show movement due to inspiration. Intensity inside lung seems to be uniform but this is only due to resampling for display purposed.

be used as input for dosimetric studies in order to estimate dose error obtained with and without breath holding. Moreover, it will also be used for building an accurate model of thorax movements in order to predict tumor and surrounding organs displacements.

Further works are ongoing to propose systematic tests in order to validate the use of breath holding techniques. We also plan to improve the model, to integrate different deformation regularization according to the local densities, to use non-rigid registration between more than two CT scans.

Acknowledgement. This work was supported in part by Elekta Oncology Systems.

\section{References}

1. S. Clippe, D. Sarrut, C. Malet, S. Miguet, C. Ginestet, and C. Carrie. Patient setup error measurement using 3D intensity-based image registration techniques. Int. J. of Rad. Onc. Biol. Phys., 56(1):259-263, 2003.

2. K.G. Gilhuijs, P.J. van de Ven, and M. van Herk. Automatic three-dimensional inspection of patient setup in radiation therapy using portal images, simulator images, and computed tomography data. Med Phys, 23(3):389-99, 1996.

3. J.W. Wong, M.B. Sharpe, D.A. Jaffray, V.R Kini, J.M. Robertson, J.S. Stromberg, and A.A. Martinez. The use of active breathing control (ABC) to reduce margin for breathing motion. Int. J. Radiat. Oncol. Biol. Phys., 44(4):911-919, July 1999.

4. E.A. Barnes, B.R. Murray, D.M. Robinson, L.J. Underwood, J. Hanson, and W.H. Roa. Dosimetric evaluation of lung tumor immobilization using breath hold at deep inspiration. Int J Radiat Oncol Biol Phys, 50(4):1091-8, 2001. 
5. M.A. Viergever J.B.A. Maintz. A survey of medical image registration. Medical Image Analysis, 2(1):1-36, 1998.

6. H. Lester and S.R. Arridge. A survey of hierarchical non-linear medical image registration. Pattern Recognition, 32(1):129-149, 1999.

7. F.L. Bookstein. Principal warps: Thin-plate splines and the decomposition of deformations. IEEE T. Pat. An.Mach. Int., 11:567-585, 1989.

8. K. Rohr, H. S. Stiehl, R. Sprengel, and W. Beil. Point-Based Elastic Registration of Medical Image Data Using Approximating Thin-Plate Splines. LNCS, 1131:297306, 1996.

9. G. Christensen, R. Rabbit, and M.I. Miller. Deformables templates using large deformation kinematics. IEEE T. Im. Proc., 5:1435-1447, 1996.

10. S. Warfield, A. Robatino, J. Dengler, F. Jolesz, and R. Kikinis. Brain warping, chapter 4: Nonlinear registration and template driven segmentation, pages 67-84. A.W. Toga, AcademicPress, 1999.

11. Y. Kawata, N. Niki, H. Ohmatsu, M. Kusumoto, R. Kakinuma, K. Mori, H. Nishiyama, K. Eguchi, M. Kaneko, and N. Moriyama. Analysis of pulmonary nodule evolutions using a sequence of three-dimensional thoracic ct images. In MICCAI'2001, volume 2208, pages 103-110, Utrecht (Netherlands), 2001. LNCS.

12. M. Bro-Nielsen and C. Gramkow. Fast fluid registration of medical images. In K.H. Hone and R. Kikinis, editors, SPIE Visualization in Biomedical Computing, volume 1131, pages 267-276. LNCS, 1996.

13. J. Barron, D. Fleet, S. Beauchemin, and T. Burkitt. Performance of optical flow techniques. In IEEE Comp. Vis. Pat. Rec. (CVPR), pages 236-242, Champaign, IL, 1992.

14. H.-H. Nagel. Displacement vectors derived from second-order intensity variations in image sequences. Comput. Vision Graph. Image Process., 21:85-117, 1983.

15. G. Hermosillo, C. Chefd'Hotel, and O. Faugeras. A variational approach to multimodal image matching. Technical Report RR4117, INRIA, 2001.

16. B. Fischer and J. Modersitzki. A super fast registration algorithm. Bildverarbeitung für die Medizin, pages 169-173, 2001.

17. J.P. Thirion. Image matching as a diffusion process: an analogy with Maxwell's demons. Medical Image Analysis, 2(3):243-260, 1998.

18. L. Fan, C.W. Chen, J.M. Reinhardt, and E.A. Hoffman. Evaluation and application of $3 \mathrm{~d}$ lung warping and registration model using HRCT images. In SPIE Medical Imaging, San Diego, CA, 2001.

19. P. Cachier, X. Pennec, and N. Ayache. Fast non-rigid matching by gradient descent: study and improvements of the "demons" algorithm. Technical Report RR3706, INRIA, 1999.

20. R. Deriche. Recursively implementing the gaussian and its derivatives. Technical Report 1893, INRIA, April 1993.

21. S. Hu, E.A. Hoffman, and J.M. Reinhardt. Automatic lung segmentation for accurate quantitation of volumetric X-ray CT images. IEEE T. Med. Im., 20(6):490498, 2001.

22. F. Maes, A. Collignon, D. Vandermeulen, G. Marchal, and P. Suetens. Multimodality Image Registration by Maximization of Mutual Information. IEEE T. Med. Im., 16(2):187-198, 1997.

23. W.H. Press, B.P. Flannery, S.A. Teukolsky, and W.T. Vetterling. Numerical Recipes in C: The Art of Scientific Computing. Cambridge University Press, second edition, 1992. 